This item was submitted to Loughborough's Research Repository by the author.

Items in Figshare are protected by copyright, with all rights reserved, unless otherwise indicated.

\title{
Computationally inexpensive methods of ion current signal manipulation for predicting the characteristics of engine in-cylinder pressure
}

PLEASE CITE THE PUBLISHED VERSION

\section{PUBLISHER}

(C) IMechE / Professional Engineering Publishing

\section{LICENCE}

CC BY-NC-ND 4.0

\section{REPOSITORY RECORD}

Gazis, Andreas, Dimitris Panousakis, Rui Chen, and Wen-Hua Chen. 2008. "Computationally Inexpensive Methods of lon Current Signal Manipulation for Predicting the Characteristics of Engine In-cylinder Pressure". figshare. https://hdl.handle.net/2134/3758. 
This item was submitted to Loughborough's Institutional Repository (https://dspace.lboro.ac.uk/) by the author and is made available under the following Creative Commons Licence conditions.

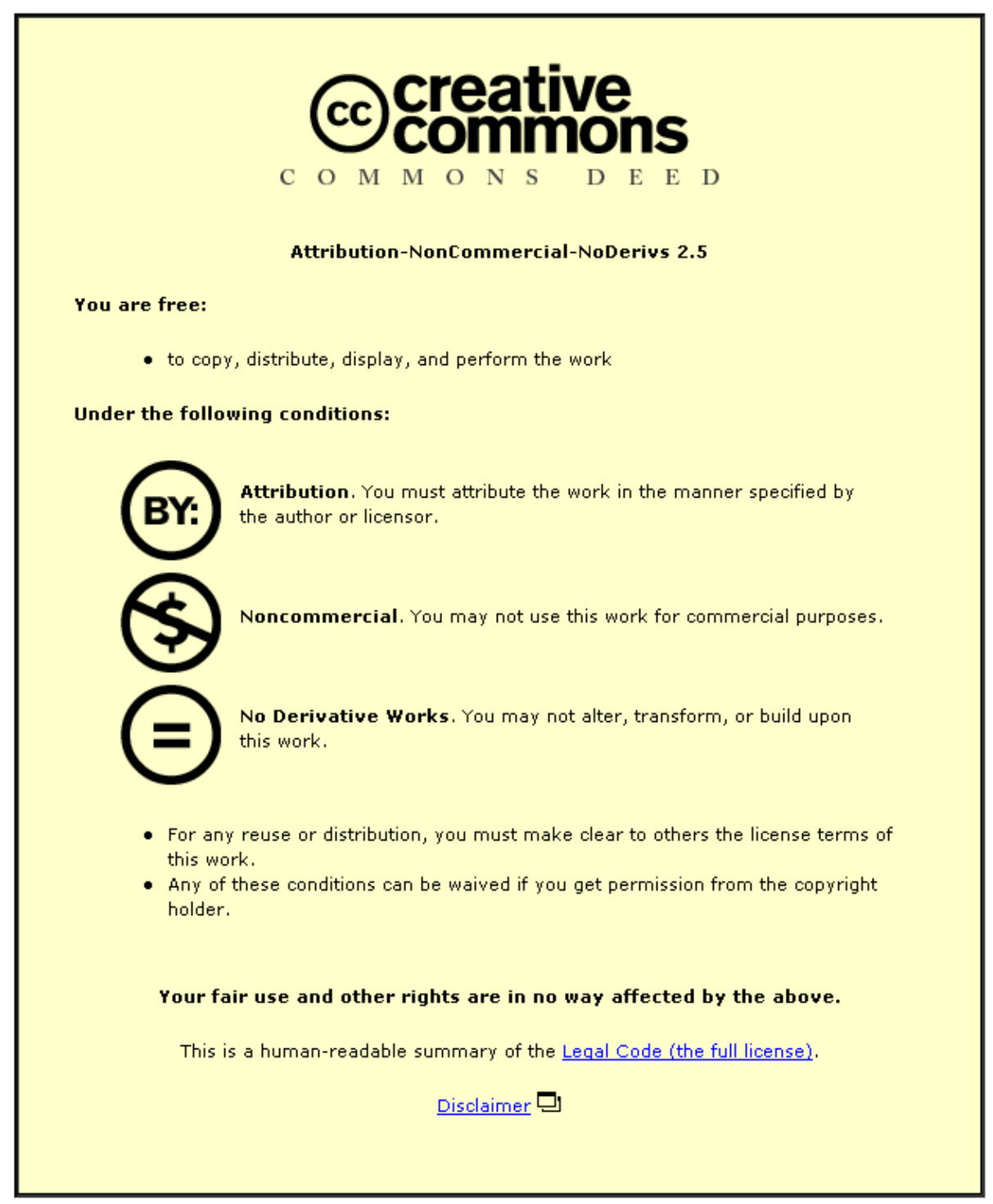

For the full text of this licence, please go to: http://creativecommons.org/licenses/by-nc-nd/2.5/ 


\title{
Computationally inexpensive methods of ion current signal manipulation for predicting the characteristics of engine in-cylinder pressure
}

\author{
A Gazis*, D Panousakis, R Chen, and W-H Chen \\ Department of Aeronautical and Automotive Engineering, Loughborough University, Loughborough, UK
}

The manuscript was accepted after revision for publication on 5 September 2005.

DOI: 10.1243/14680874JER04005

\begin{abstract}
Recent research on the use of ion current has focused on matching the characteristics of the in-cylinder pressure, thus avoiding the use of a pressure transducer. This paper explores techniques of calculating these pressure characteristics through the use of simple and computationally inexpensive artificial neural networks. Two neural networks are presented to deduce the in-cylinder pressure from ion current measurements, where one is used to predict the characteristics directly and the other is used to calculate the in-cylinder pressure curve. Experimental results show that both networks give satisfactory results for different purposes. Some engineering implementation issues and the further improvement of the developed techniques are discussed.
\end{abstract}

Keywords: ion current, in-cylinder pressure, artificial neural networks

\section{INTRODUCTION}

In the race for more efficient engines, conventional means of control have to evolve in order to provide the finesse required. A modern engine is a greatly computerized entity with a host of sensors checking a variety of aspects of its operation. However, largely owing to the extreme conditions that it contains, the cylinder itself is usually not equipped with any sensors monitoring it directly. Still, knowledge of what happens inside the cylinder is highly desirable for a modern control system. Most manufacturers try to deduce relevant information indirectly through knock sensors, lambda sensors, airflow sensors, etc., and conditions inside the cylinder are predicted from models based on these sensors' outputs, rather than on direct measured evidence. Thus these are not as suitable for the task as an in-cylinder sensor.

Cylinder pressure is one of the most important variables in monitoring engine performance. The most obvious solution is to install a pressure sensor in each cylinder. This, however, is impractical because of the prohibitively high cost and question-

\footnotetext{
* Corresponding author: Department of Aeronautical and Automotive Engineering, Loughborough University, Loughborough, Leicestershire LE11 3TU. email: a.gazis@lboro.ac.uk
}

able long-term performance of such a device. Thus pressure sensors are used for research purposes, a target to be met rather than a solution in themselves.

Ion current has long been investigated as a combustion diagnostic tool [1-5]. It works as follows. The prevailing conditions during the combustion causes ionization of the gases inside the cylinder. This ionization occurs mainly in two phases. The first phase occurs during combustion as fuel reacts with oxygen. It is defined as the chemical phase. The second phase occurs as the already burnt gases are compressed by rising pressure created by the fuel farther away from the spark plug combusting. It can therefore be defined as the thermal phase. By applying a voltage through these gases, a current will be observed. It is up to the engine controller then to decide what kind of information can be deduced from this ion current signal [6-7].

The potential of ion current has been researched and used in production as a means of misfire detection (a task that it is well suited for as a misfire produces no ion current) and knock detection [8-10]. More research has focused on the estimation of airfuel ratio (AFR) from manipulation of ion current signals [11-16] and how to incorporate it into a closed-loop control system [17-22].

Recent research has gone further, trying to repro- 
duce in-cylinder-pressure profile characteristics from ion current data [23-28]. If successful, the advantages are obvious. The high cost of the pressure sensor can be bypassed with just the cost of relatively cheap electronics.

The most obvious way to apply a voltage inside a cylinder is to use two already existing electrodes, the spark plug tips. This approach has some inherent problems. In most engines there is one plug per cylinder which has to generate a spark as well as to measure ion current. Because the spark-generating voltage is substantially higher than the typical voltages applied in the ion-current-measuring circuit, the latter has to be protected somehow. This has been accomplished for example by either measuring from the low-voltage side of the ignition circuit or by switching the measuring circuit in and out of the high-voltage side. Both these approaches complicate matters, the former because of increased noise and bandwidth filtering by the coils, and the latter because of the intricacy of such a dedicated circuit. Furthermore, all approaches that measure ion current signals from the ignition spark plug suffer from the fact that no meaningful information can be gathered until the ignition circuit has been fully discharged. As a result, the initial stage of the combustion, which corresponds to the chemical phase of the ion current signal, cannot be recorded $[\mathbf{2 9}, \mathbf{3 0}]$.

Another significant problem of measuring ion current through the firing spark plug is that, since the initial stage of the combustion cannot be well measured, the best signal is derived from the ion current signal's thermal phase. This, however, becomes less significant with reduced load and can disappear for load settings less than 75 per cent, thus severely limiting the usefulness of the ion current signal.

In order to address these issues, ion current sensing from dedicated sensors can be employed (remote sensing), introducing significant advantages. On the technical side, signal quality is greatly improved since data acquisition (DAQ) does not need to be interrupted. This continuous measurement allows for acquisition of ion current data throughout the combustion process. This enables a much a greater volume of information to be extracted from the signal at higher signal-to-noise ratios. Since this signal potentially holds much information to be extracted, sophisticated signal processing strategies need to be employed.

On the cost side, this approach offers the opportunity for simpler, more robust and therefore more cost-efficient designs for the measuring circuit, since there is no consideration of coupling with the ignition circuit to be taken into account.
Finally, signal quality is greatly improved using remote sensing, thus permitting true cycle-to-cycle engine diagnosis and control. Thus, the need for averaging is eliminated, which reduces computational and time requirements.

It might, at first, seem as a significant complication to introduce dedicated ion sensors on mass production engines. However, companies that already offer head gaskets with multiple ion collectors exist. This makes modifications to the cylinder head and engine block unnecessary [31, 32]

Thus, this research focuses on the examination of the potential of ion current based mainly on the benefits of better signal quality, cheaper electronics, and use of computationally inexpensive signal-processing algorithms that are made possible through remote sensing.

After examining the relations between the ion current and cylinder pressure and investigating the characteristics of these two signals, artificial neural network (ANN) techniques are used to deduce the cylinder pressure information from the ion current measurement and knowledge of the operating conditions. To reduce the implementation difficulties, a simple and computationally inexpensive adaptive linear element (ADALINE) type of network is chosen for this purpose. Then the networks are trained with a number of data sets for different operating conditions. The trained networks can deduce the cylinder pressure information required for engine monitoring and cycle-to-cycle closed-loop engine control from the ion current measurement and the operating conditions such as speed and load. To verify the effectiveness of the proposed techniques, experiments are designed to compare the deduced pressure yielded by the networks using ion current measurement with the actual in-cylinder pressure.

ANNs have been used before to interpret ion current signals. These are most commonly of the perceptron type, a standard ANN for general usage. ADALINEs are networks widely used in industrial applications too; however, their use for ion current interpretation has not been witnessed by the present authors in the relevant literature. ANNs offer a promising tool for this type of task, however, for the reasons outlined in section 4, ADALINEs are chosen as the best candidate.

\section{EXPERIMENTAL SET-UP}

The engine employed in the investigation is a singlecylinder four-stoke research engine. The basic parameters are listed in Table 1. It features variable 


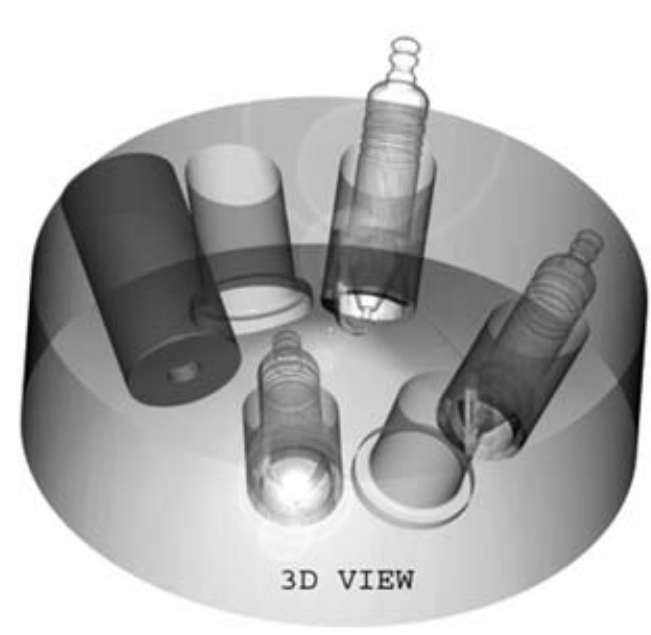

(a)

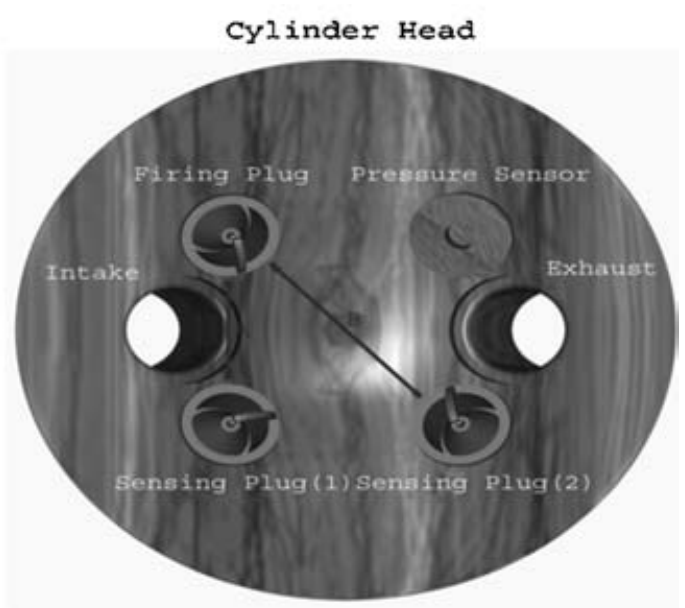

(b)

Fig. 1 Configuration of cylinder head

Table 1 Engine parameters

\begin{tabular}{ll}
\hline Bore & $80 \mathrm{~mm}$ \\
Stroke & $100 \mathrm{~mm}$ \\
Capacity & $0.503 \mathrm{l}$ \\
Inlet valve opening & $12^{\circ}$ before top dead centre (BTDC) \\
Inlet valve closing & $64^{\circ}$ after bottom dead centre \\
Exhaust valve opening & $64^{\circ}$ before bottom dead centre \\
Exhaust valve closing & $12^{\circ}$ after top dead centre (ATDC) \\
Compression ratio & $4.5: 1-13: 1$ \\
Ignition timing & $55^{\circ} \mathrm{BTDC}, 20^{\circ}$ ATDC \\
Maximum speed & $4500 \mathrm{r} / \mathrm{min}$ \\
Maximum power & $16 \mathrm{bhp}$ \\
\hline
\end{tabular}

compression ratio, variable ignition timing, variable AFR, variable valve timings, and four access points on the cylinder head for up to three spark plugs and one pressure sensor to be located.

Figure 1 shows the configuration of the cylinder head. There are four access points. One was fitted with a firing spark plug (bottom left). The next two were equipped with two sensing spark plugs (top and bottom right). The ion current was detected by these two remotely located spark plugs. The fourth access point was fitted with a pressure transducer.
The tips of the sensing spark plugs were assigned as the positive electrodes while the spark plug body together with the rest of the combustion chamber were used as the negative side. This was convenient since the original engine polarity was preserved.

The voltage source shown in Fig. 2 produces a voltage of $5 \mathrm{~V}$, to the DAQ, for an infinite resistance between the ion sensor electrodes. This voltage reduces in an inversely proportional manner to any measured ion current signal, building in DAQ protection.

In order to reveal the true ion current signal, the measure voltage signal has been re-inverted by a voltage divider before feeding into the computer DAQ board. The DAQ sampling rate was one sample per $2^{\circ}$ of crank angle (CA) at all engine speeds, thus making it easily implemented for mass production purposes. The ion current signals from the two sensing plugs were fed into the DAQ board, as shown in Fig. 2. Therefore, an ion current signal from either sensor or their sum can be presented and analysed.

Use of this set-up presents the whole flame-front

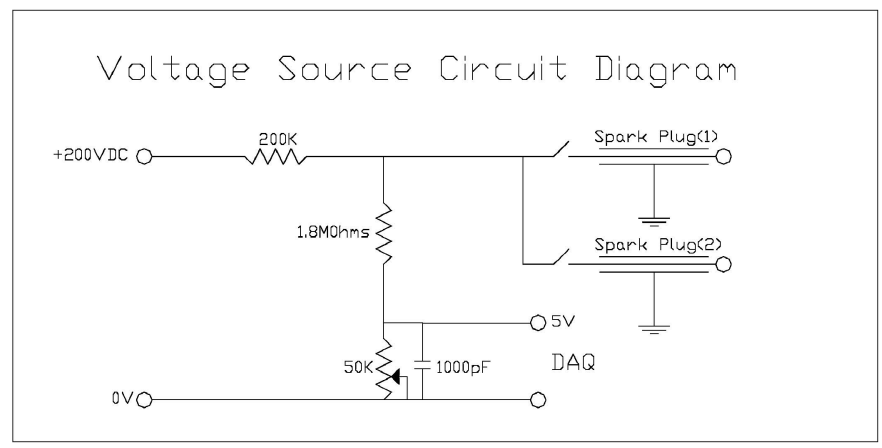

Fig. 2 DC voltage source 

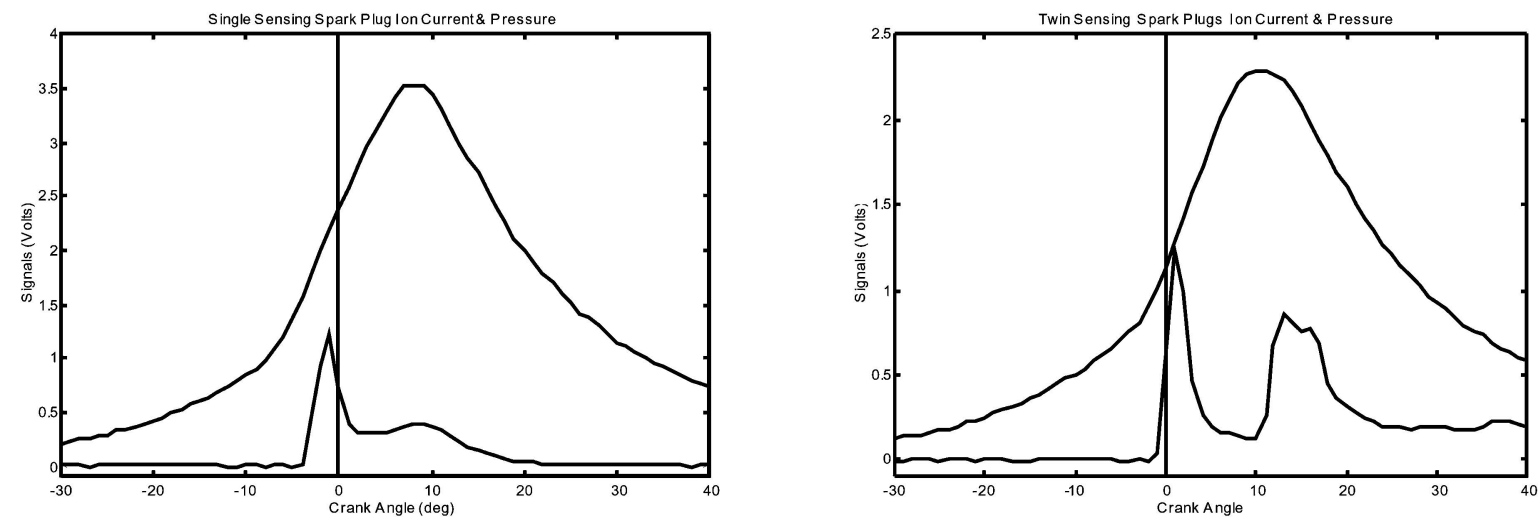

Fig. 3 Examples of pressure and ion current logs

profile as measured while it sweeps past the plugs as opposed to measuring the back of it as it recedes from the plug in a typical set-up.

Examples of pressure and ion current logs using one or both sensors can be seen in Fig. 3. Results obtained using both sensors contain more information, thus generally produce higher correlations. Throughout the rest of the paper, only results obtained using both sensors are discussed.

\section{SIGNAL INTERPRETATION}

The experimental data consist of two signals. The first is the in-cylinder pressure, measured from the pressure sensor. The second is the ion current signal measured from the two measuring plugs. Both of these signals are sampled every $2^{\circ} \mathrm{CA}$. Figure 4 shows them together for a typical combustion event.

The leftmost vertical line indicates inlet valve closing (IVC). The next line indicates ignition timing

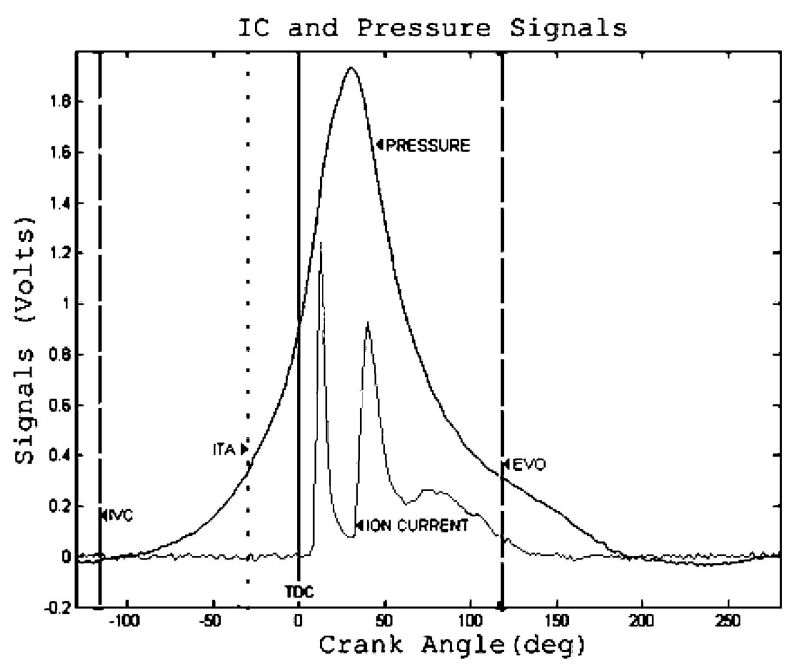

Fig. 4 Pressure and ion current signals advance (ITA). The line at $x$-axis zero indicates top dead centre (TDC). The rightmost line indicates exhaust valve opening (EVO).

\subsection{Measurants}

The pressure signal is simpler than the ion current signal. In order to describe it numerically, four characteristics or 'measurants' are extracted for every combustion event. Figure 5 shows a typical pressure curve and associated measurants.

The measurants for the pressure curves are as follows:

(a) the peak pressure position ( $x$ axis);

(b) the peak pressure magnitude ( $y$ axis);

(c) the width of the curve at half its height;

(d) the area of the curve between IVC and EVO.

The ion current is a much more interesting signal. Because it is measured from two different plugs at different distances from the firing plug, there are two

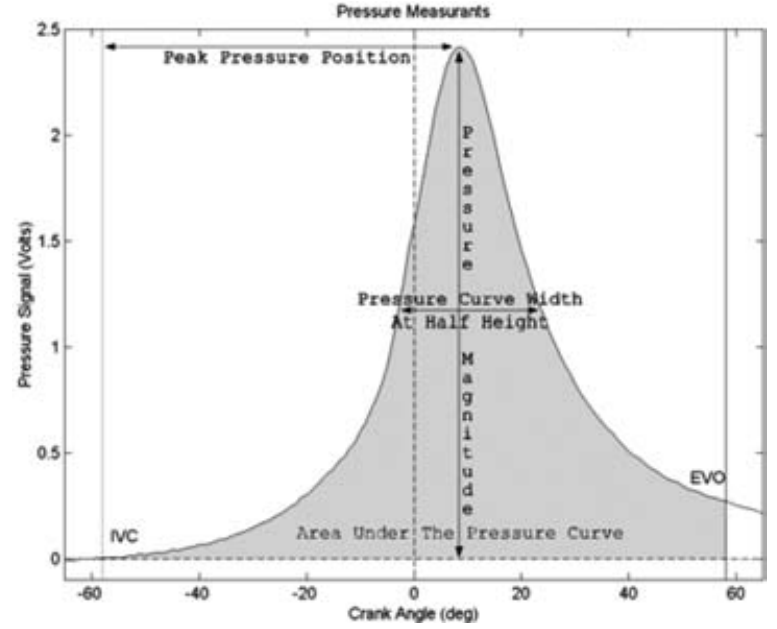

Fig. 5 Typical pressure curve and associated measurants 
distinct spikes. This is because the flame front will sweep through the measuring electrodes at slightly different times. After the second spike there is the hump associated with the post-flame phase.

One point to consider is how signals from two sensing plugs create a single trace. The circuit used simply adds up the two signals and so what is seen is the sum of the ion current on both plugs. This would lead to the expectation that the second spike is heavily affected by the post-flame phase, since both measuring plugs are registering at that time (whereas, for the first spike, the flame has only reached the first plug), degrading its information content. This, however, is not the case. Figure 6 shows a close-up of an ion current trace with only one measuring plug and the engine operating highly throttled so as to minimize the post-flame hump. These are the conditions that create the most problems when measuring from the firing plug, as is the usual practice. Hellring et al. [24] mentioned that 'the post-flame peak essentially vanishes if the load is less than 20 per cent of the maximum load'. It is evident from Figs 3 and 4 that the first spike only lasts for about $10^{\circ} \mathrm{CA}$. The post-flame phase is missing completely since ionization due to compression of the gases is low owing to the low-load conditions. Employing remote sensing eliminates the dependence on the post-flame signal. Employing two remote sensors adds further signal information.

The third phase (thermal post-flame hump) is a less-localized lower-magnitude event. Although the time window available for post-flame signal acquisition is reduced when using the sum of the signal from the two sensors, the results obtained are superior.

As in the pressure curve, some measurants are needed to describe the ion current signal for a combustion event. In the case of ion current there are 13

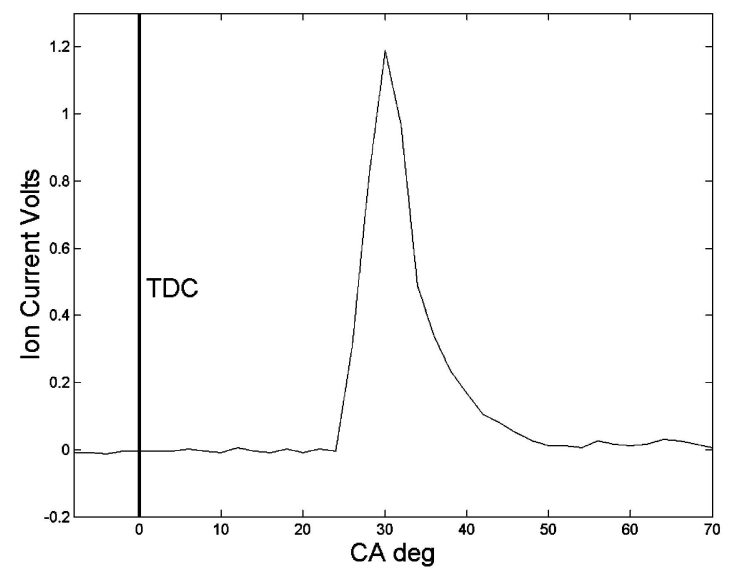

Fig. 6 Single plug trace such measurants. A typical ion current signal and its associated measurants are shown in Fig. 7.

The measurants for the ion current signals are as follows:

(a) the $x$-axis position of the start of the signal;

(b) the $x$-axis position of the first spike;

(c) the $x$-axis position of the second spike;

(d) the magnitude of the first spike;

(e) the magnitude of the second spike;

(f) the slope of the curve between the signal start and the first spike peak;

(g) the slope of the curve between the first spike peak and the minimum between the spikes;

(h) the slope of the curve between the minimum between the spikes and the second spike peak;

(i) the slope of the curve between the second spike peak and a point on the curve at the same $x$-axis distance as used for the previous slope;

(j) the area under the first spike;

(k) the area under the second spike;

(l) the area under the post-flame section;

(m) The $\mathrm{x}$-axis position of the end of the signal.

After comparative tests, these measurants were chosen as carriers of adequate information to describe this signal. Of these, the various $x$-axis positions proved the most useful, since they are related to flame development. However, all selected measurants contribute to increasing the accuracy of the results.

\subsection{Feature relations}

Given the measurants extracted, the easiest way to look for relations is to plot them against each other. Ideally, a strong relation between an ion current

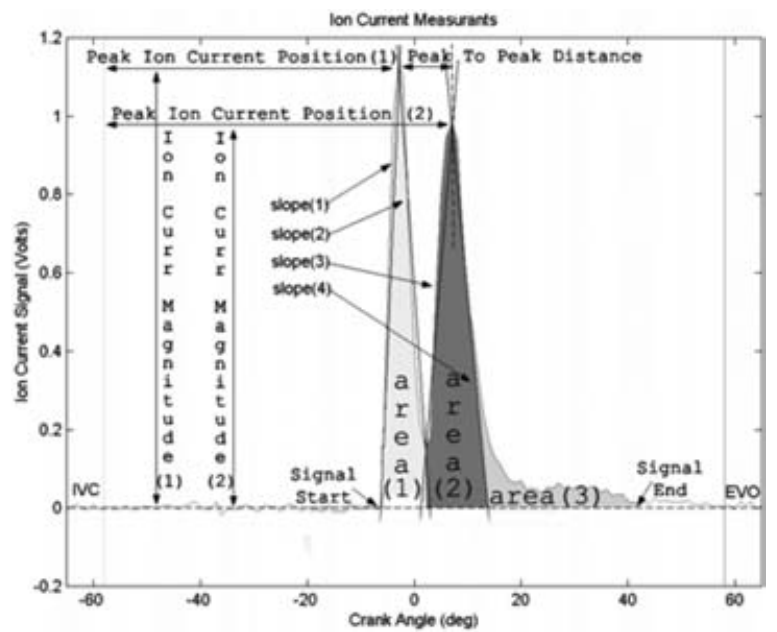

Fig. 7 Typical ion current signal and associated measurants 
measurant and one or more pressure measurants will settle the case in favour of ion current. However, it is not that simple. There are conclusions to be drawn by averaging over a number of cycles, which is the technique used routinely in treating such signals. These, however, are not helpful when developing a tool; this should, in practice, be able to help to control cycle-to-cycle engine operation, which is the aim of this investigation.

Figures 8 to 10 show some cases of strongly related measurants. Ion current measurants are on the $x$-axis; pressure measurants are on the $y$-axis. These data are logged over a varying compression ratio loop, therefore each data point batch corresponds to a compression ratio between 4.5:1 and 11.2:1.

Figures 8 and 9 are matches for the peak pressure position with the positions of the first and second ion current spikes respectively. It shows good correlation with both measurants and that a delayed combustion event results in a late pressure peak position. Another point to note is that delayed combustion

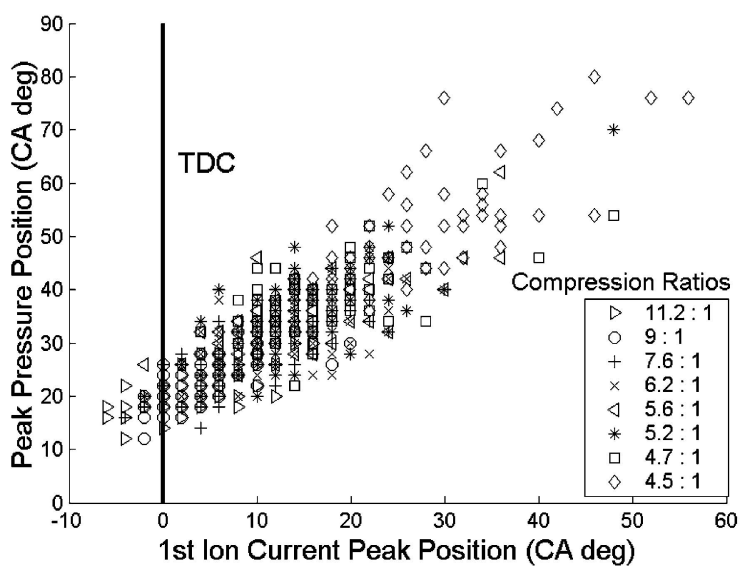

Fig. 8 Relationship between the first peak ion current and the peak cylinder pressure position

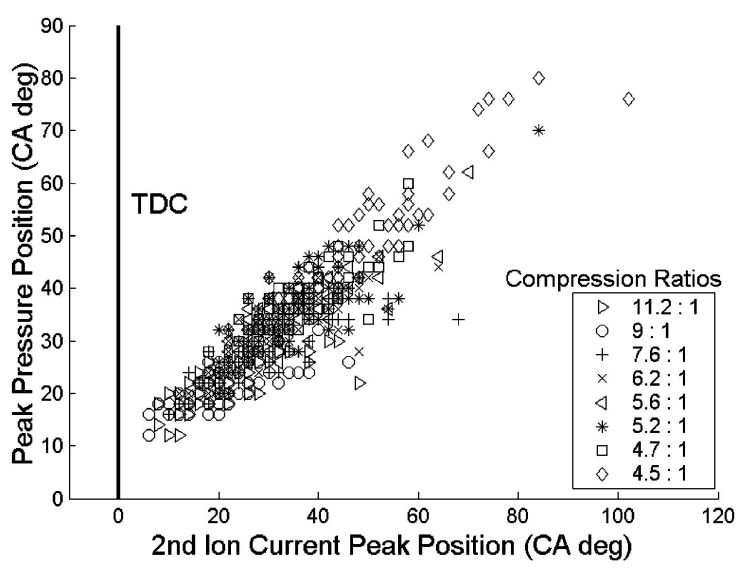

Fig. 9 Relationship between the second peak ion current and the peak cylinder pressure position

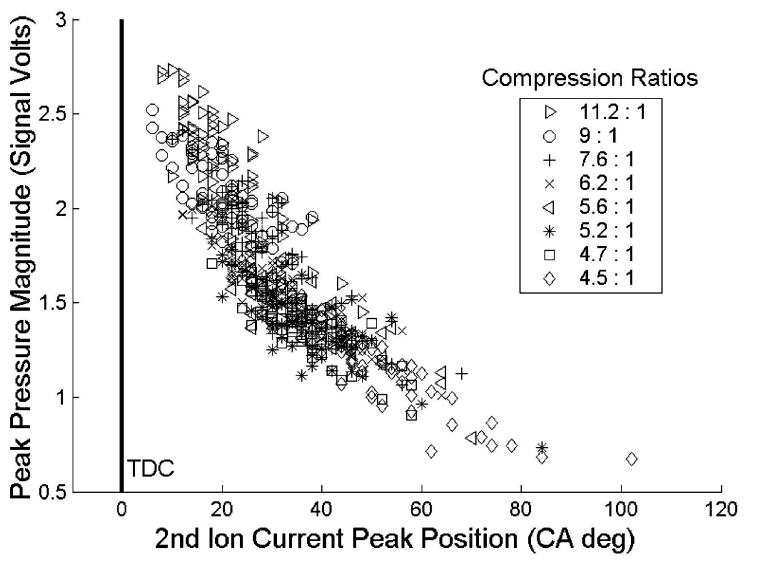

Fig. 10 Relationship between second peak ion current and the peak cylinder pressure

results in a greater uncertainty in the peak pressure position. This arises because delayed combustion is more unstable, resulting in higher cycle-to-cycle variation.

Figure 10 is a match between peak pressure magnitude and the position of the second ion current spike. It can be seen that the second peak ion current position has a strong relationship with the peak cylinder pressure magnitude, too. When the second ion current spike occurs late, the flame reaches the second sensing plug late, which indicates late combustion. Therefore the peak cylinder pressure magnitude is reduced.

Although the relationships between the first and second ion current spikes with the combustion event are strong, as can be seen in Figs 8 to 10, there is an uncertainty of the order of $10^{\circ} \mathrm{CA}$ relating to the peak pressure position and of the order of $0.4 \mathrm{~V}$ relating to the peak pressure magnitude. These uncertainties are not acceptable for engineering implementation of the use of the ion current as an alternative means of measuring the cylinder pressure. For this reason, a more sophisticated strategy has to be developed and employed for ion current signal interpretation in order to improve its correlation with the pressure signal.

\section{PREDICTIONS}

\subsection{Artificial neural networks}

ANNs are a good candidate for tackling this kind of problem $[14,22,23]$. These are computational constructs, used in a variety of applications for dealing with complicated inputs. The notion behind them is loosely modelled on real neural networks. Each artificial neuron is a node that takes a number of inputs. 
These are weighed and then summed as illustrated on the left-hand side of Fig. 11.

The idea is that an input with a strong relation to the output will have a relatively large weight associated with it. Thus, fluctuations in important inputs will result in significant changes to the weighted sum.

Finally, the weighed sum is then passed through a transfer function to give the final output. There are various traditionally used transfer functions, some of which are shown in Fig. 12.

Their role is to 'summarize' the inputs of the neuron into a value. The simple form of Fig. 12(a) is a step function which basically translates to on and off states for the artificial neuron. Figures 12(a) to (d) are variations on the same theme, making a smoother transition so that information is not lost in the grey area where the weighted sum does not translate clearly into the on or off areas.

A collection of artificial neurons is what is termed the artificial neural network as illustrated in Fig. 13. The inputs are taken in by the input layer of artificial neurons. These are then processed through successive layers until they reach the final 'output' layer.

The main tasks when designing an ANN is to decide upon the architecture best suited to the task, e.g. number of layers and type of transfer functions, and then to tune its parameters, e.g. the various weights. There are many different types of training

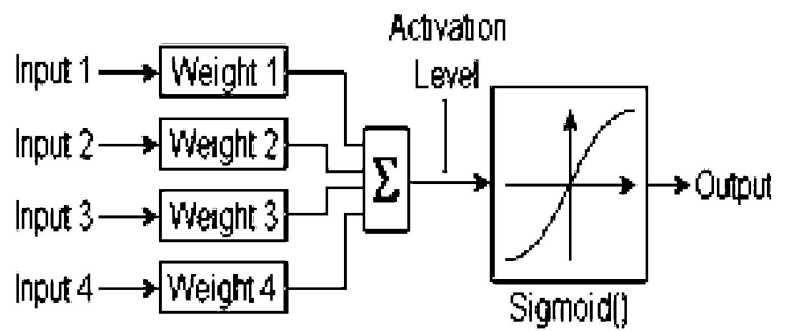

Fig. 11 Schematic representation of an artificial neuron

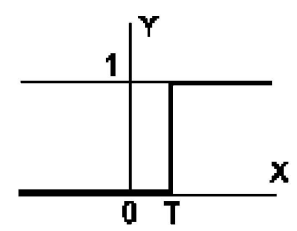

a)

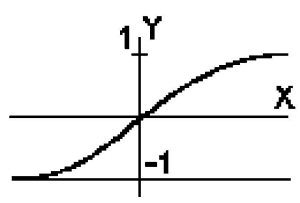

B)

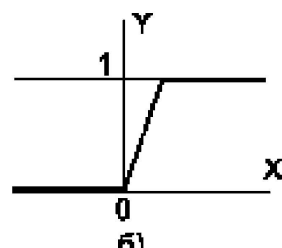

6)

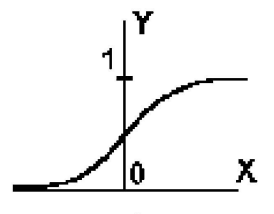

Г)
Fig. 12 Sample transfer functions

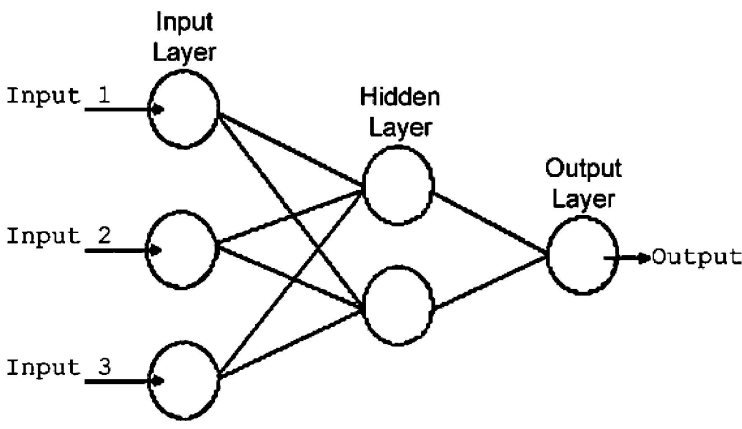

Fig. 13 A simple ANN

algorithm for this task. Most of these are recursive where a network is presented with successive sets of inputs; small alterations are made each time according to its response compared with the desired values until at some point tuning is decided to be adequate for the task.

In the case of this experiment, the following considerations are taken into account.

First, the network has to be as uncomplicated as possible. If the model is to be usable on a cycle-tocycle basis by an engine controller, all computations have to be completed sufficiently rapidly to provide timely results for the next cycle for every cylinder. The simpler the network that does this, the less demand there is on the signal-processing electronics that will carry out those computations.

Second, the network or some further algorithm behind it must produce continuous output. This is important as the measurants are numbers that cannot be represented by an on-off state. To code for such states using step transfer functions requires a large amount of output artificial neurons for each output.

Third, the network must be easy to train with a reasonably small amount of data. In engineering applications, it is impractical to require a vast amount of data to train a neural network owing to the cost and time needed to collect these. The goal was to use as few as 70 combustion events per engine operating condition.

There are several network families to choose from. Keeping the above points in mind, ADALINEs were chosen. These ADALINEs are among the classic types of ANN. An ADALINE neuron takes a weighted sum of its inputs but, instead of passing it through a transfer function, sends it straight to its output. This is also helpful for producing continuous output since a single output artificial neuron can produce any real value. The main limitation of ADALINEs is that they will tackle linear relations but will not be very useful beyond these. Looking at the data in Figs 8 to 10, both linear and non-linear relationships are 
indicated. However, the window of engine operating conditions tested is strongly exaggerated compared with normal operation. Despite this and the fact that linear approximations were used, the obtained results are well within the scope of closed-loop engine control implementation. In addition, the range of operating conditions spanned by a production engine is such that the data produced would be in relations even more adequately modelled as linear.

Finally, for training purposes, ADALINEs can be trained using the least-mean-squares (LMS) algorithm. This is a very important consideration given the low-data-volume requirement. The strength of the LMS algorithm lies in that it competently handles this case where the data are limited. The way that LMS trains the network is as follows: given a set of inputs and a set of desired outputs, the error is defined as the difference between the actual output and the desired output. LMS minimizes the average of the sum of the square of these errors. Since this is a quadratic function, it will have at most one minimum. Thus, for a given set of inputs, LMS will tune the network so that their averaged squared errors are minimized. In this research, two sets of networks have been developed and tested. To test these, some data sets were set aside and used afterwards to assess performance under unknown inputs.

\subsection{Measurant-predicting network}

The first network is the smallest in terms of artificial neurons. It is a layer of four artificial neurons (ADALINES are single-layer networks), each of which takes the 13 ion current measurants as inputs and produces one of the predicted pressure measurants as an output. Each input is multiplied by a weight factor and all weighted inputs are then summed into what becomes the output.

Figure 14 shows the network architecture. The 13 ion current measurants are the set of circles at top

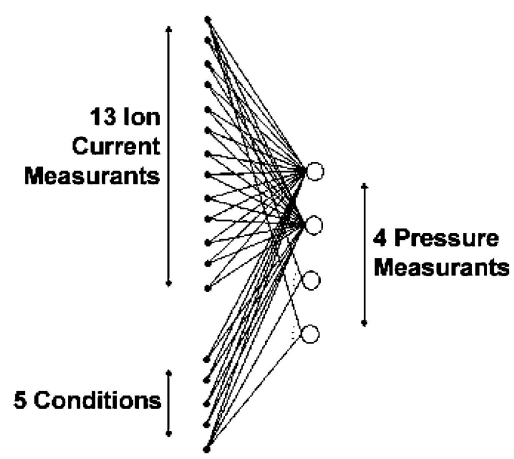

Fig. 14 Schematic representation of the network left. The set under them, labelled 5 Conditions is a string of numbers (constant for each input file) that describes the operating conditions of the engine at the time. These conditions are throttle position, engine speed, engine load, AFR, ignition timing advance, and compression ratio.

Training is carried out as follows. When data were logged, one of the aforementioned operating conditions was varied and a number of files logged for various values of that condition with everything else kept the same. This created 'families' of files, the compression ratio family, the ignition timing family, etc. Of these families, one member is selected to be the 'test set', the set of values to be shown to the network after training to test performance. The rest of the family is then used to train the network. Thus, when testing the network, it is given values not encountered during its training. By choosing the test set to be somewhere in the 'middle' of the variable condition range, the network arrives at the correct results since these lie within its training window. The results of this process can be seen in Figs 15 to 18 .

The entries on the $x$ axis are the measured values of the measurant, and the entries on the $y$ axis are

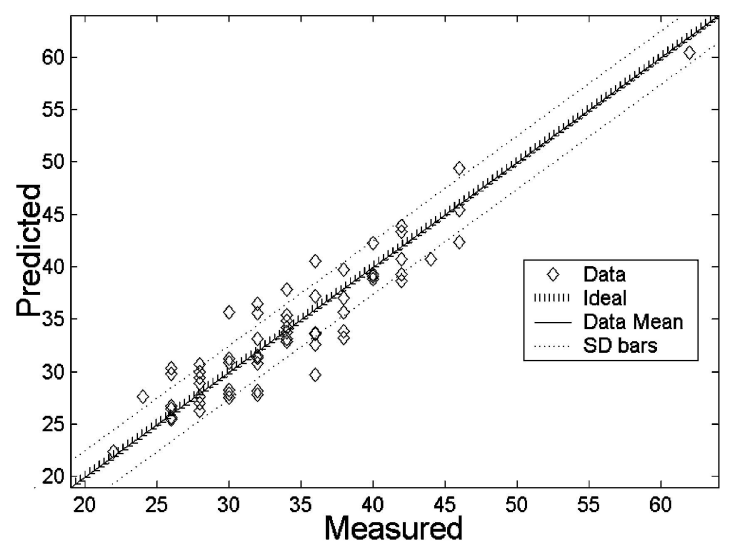

Fig. 15 Predictions for peak pressure position

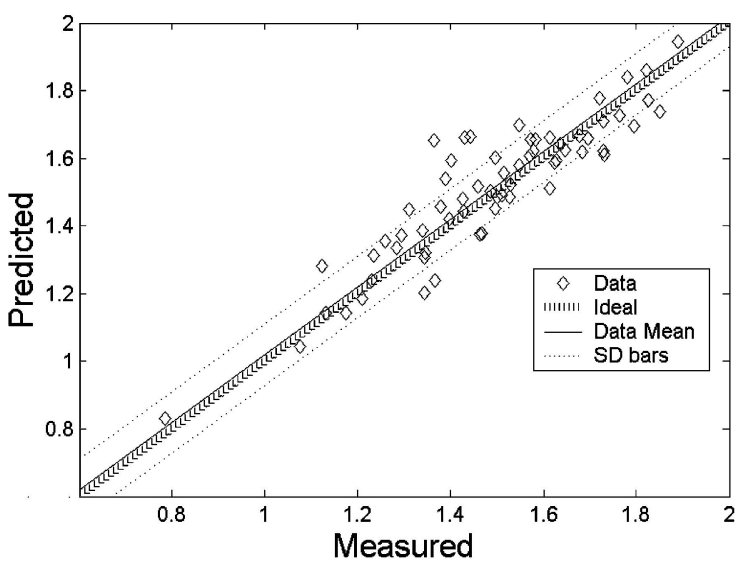

Fig. 16 Predictions for peak pressure magnitude 


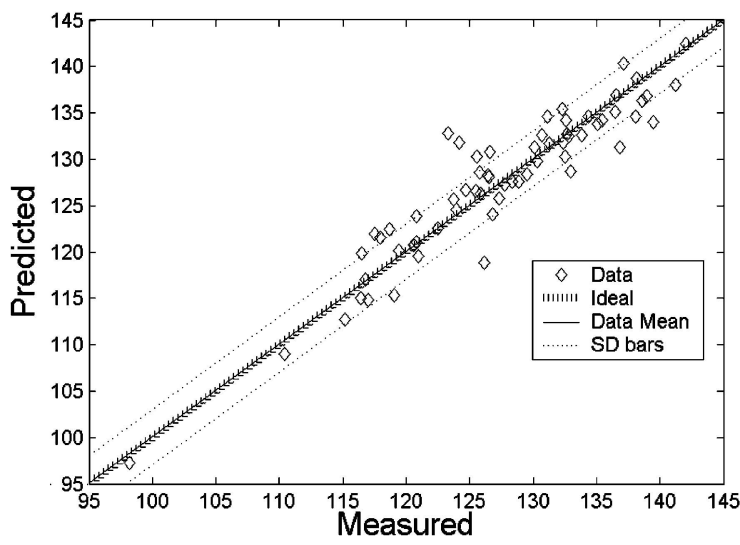

Fig. 17 Predictions for pressure curve area

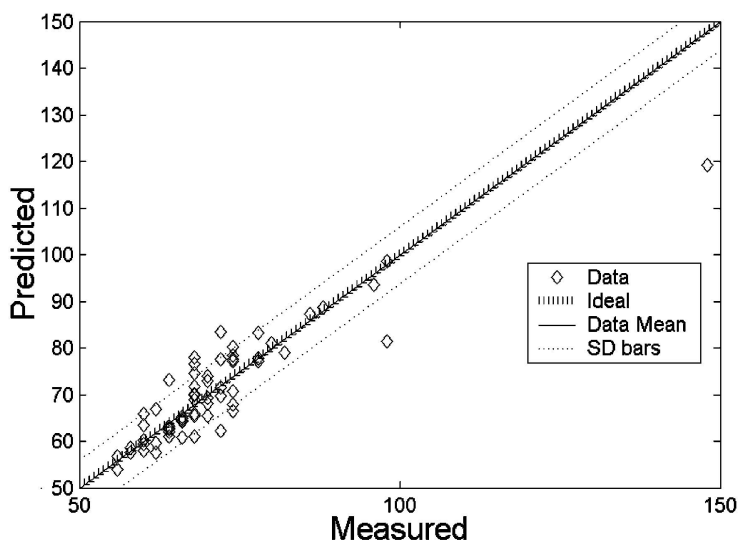

Fig. 18 Predictions for pressure curve width

the predicted values. Thus, a perfect prediction would plot a diagonal line. This is the 'ideal' line mentioned in the keys and is used for reference. The solid line indicates the data mean. It often coincides to a great extent with the 'ideal' line, which indicates a good match between measurements and predictions. The two outer dotted lines indicate the standard deviations (SDs) of the data. In these four figures, the network performs so well that the mean of the predictions and the diagonal are almost identical. The only figure in which they can be seen separately is Fig. 16.

Of the four outputs of the network, the most important are the peak pressure position and peak pressure magnitude which give the $x$ and $y$ coordinates respectively of the pressure curve's peak. Figure 15 shows the predictions for peak pressure position. The mean of the predicted values is shifted by $0.062^{\circ}$ from the mean of the actual values and the standard deviation of the predictions is $2.55^{\circ}$. A similar degree of accuracy was obtained for the rest of the in cylinder pressure measurant predictions. Given that the ion current signal is sampled every $2^{\circ}$ CA, this result is satisfactory.
The predictions in these figures are calculated from a mixed training set. The families used are the compression ratio family with various compression ratios and the speed and load families with various speeds and loads. The compression ratios for the first family are 11.2:1, 7.6:1, 6.2:1, 5.2:1, 4.7:1, and 4.5:1. The speed-load settings for the second family are 33 per cent at $1400 \mathrm{r} / \mathrm{min}, 75$ per cent at $1400 \mathrm{r} / \mathrm{min}$, wide-open throttle at $1400 \mathrm{r} / \mathrm{min}, 33$ per cent at $1750 \mathrm{r} / \mathrm{min}$, and 75 per cent at $1700 \mathrm{r} / \mathrm{min}$. The test set is a member of the compression ratio family with a compression ratio of 5.6:1. Ignition timing for these was kept constant at $30^{\circ}$ BTDC.

What is worth noting is that the network gives better results when the families are mixed together than when the training is performed on each individual family. This is a satisfactory result, demonstrating how the network can interpolate and select the right predictions for the test set, the operating conditions that it has not encountered at all during training.

\subsection{Curve-predicting network}

Given the satisfactory performance of the simple ADALINE in tackling the 13-input by four-output measurant predictions, a new network was tested predicting the whole pressure curve. Again an ADALINE was used, but this time with the whole ion current signal as the input and the whole pressure signal as the output. More specifically, each cycle is examined between IVC and EVO. Given the sampling rate and the valve timing, this gives a data string of 117 elements for both ion current and pressure. Thus the network consists of 117 artificial neurons, each of which is connected to all inputs and which produces one output, corresponding to a point on the predicted pressure curve. Figure 19 shows the measured and predicted pressure curves resulting from

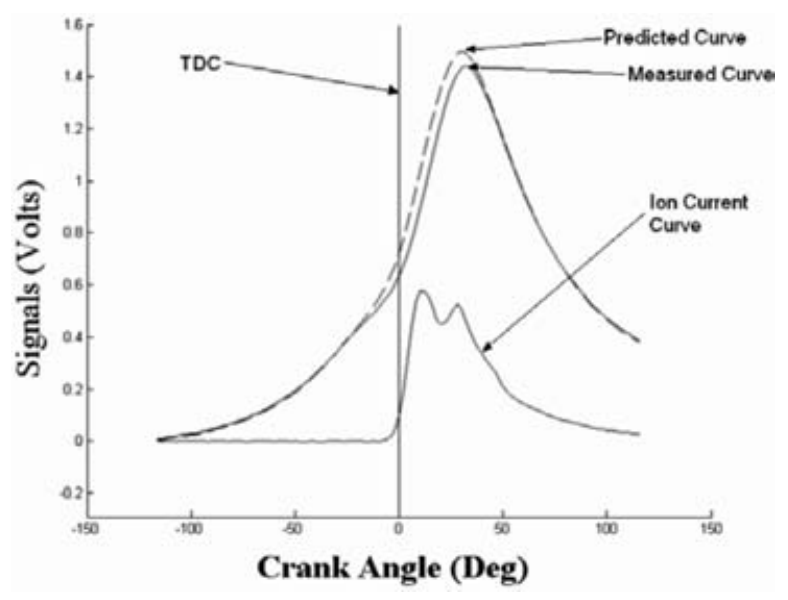

Fig. 19 Averaged actual and predicted pressure curves 
this network averaged over the whole of the test set. The training set and test set are the same as for the previous network.

Again, the averaged ADALINE follows the target quite closely even though the compression ratio of the test data has never been encountered in its training. However, the measurants extracted from the predicted curves are not as good as for the previous network. Figure 20 shows the actual and predicted values for the peak pressure position. The mean of the predicted values is shifted by $1.69^{\circ}$ (compared with 0.062 for the previous network) and the standard deviation is $5.45^{\circ}$ (compared with 2.55 for the previous network).

Similar results are true for the rest of the measurants, with uncertainty increasing roughly twofold compared with the previous network.

This network is created with the task of matching the curve point to point. The objective of training the network is to minimize the average difference between the actual and predicted curves, which may result in the fact that the error between the predicted and actual in-cylinder pressure peak positions is not minimum.

\section{DISCUSSION}

The results reported here showed how ion current data can be treated using simple techniques to predict various features of the in-cylinder pressure. Of the two networks presented, the most likely to be suitable for the task of engine control is the first (measurant prediction). This is by far the cheapest computationally and is specialized in predicting the most important aspect of the pressure curve, the location of its peak. This should be a welcome result for the further development of engine control systems striving to employ fast and simple algorithms

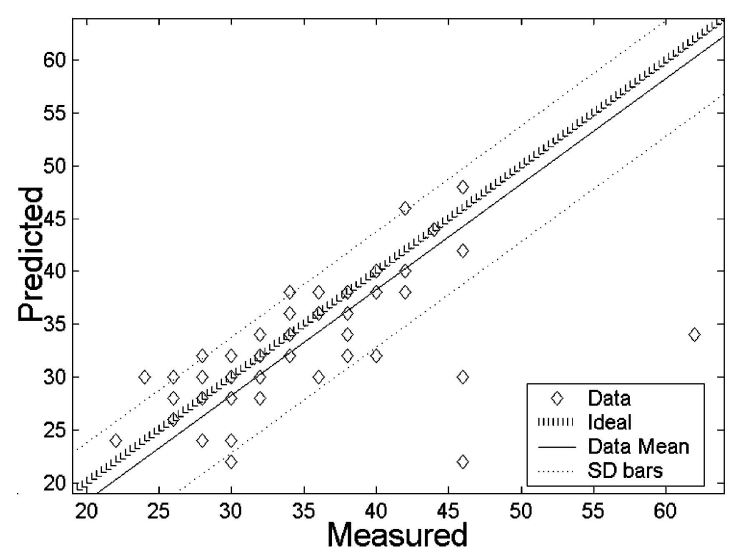

Fig. 20 Predictions for pressure curve peak position for changing engine parameters on cycle-to-cycle timescales. One drawback of this approach is that, even though the network used is itself extremely simple, its inputs are the results of some data processing since the ion current measurants used are themselves the product of various operations. Even though these operations are well within the capabilities of modern electronics, they can be more expensive computationally than the operation of the network itself, a factor that will have to be taken into account when designing such a system.

Another feature to take into account regarding measurants is the $y$-axis data of the ion current signal. All the measurants strongly affecting the pressure curve results were those on the ion current signal $x$ axis, in other words related to the timing, and not the magnitude, of the ion current events. Figure 4 shows an ion current signal. What should be noted is the sharp slopes leading up to the two peaks. In some cases, there could be two to four data points from bottom to top of the ion current spike. Therefore, the ion current signal might contain frequencies which are too high for our sampling rate. This might be an additional factor to explain why ion current magnitudes never showed any strong relation to any pressure measurants in the measurant-to-measurant plots.

The ADALINEs employed in this research manage their predictions based mostly on $x$-axis ion current measurants. It might be possible that higher sampling rates can improve the results further as the significance of $y$-axis ion current measurants will be taken into account. However, other researchers [29] have pointed out that large cyclic fluctuations are a typical problem with ion current measurements anyway. Thus, by not relying on ion current magnitude but rather on ion current timing (made easy by measuring from the remote plugs) this problem can be avoided to some extent.

Apart from the most important position of the peak of the pressure curve an ADALINE has been found to be able to tackle quite competently the task of predicting the pressure curve itself. Normally, such tasks are best left to more specialized (and more computationally expensive) tools such as radial basis functions. An important point to consider in this second network is that the input is passed to it 'raw' as it were, with no need for preprocessing to extract ion current measurants. There is a cost to pay at the output as pressure measurants are extracted from it but it is a much simpler task as there are only four measurants involved in the pressure curve as opposed to 13 in the ion current signal. However, this 
network is not as effective in locating the peak of the pressure curve.

It might seem to be a logical step to design an ADALINE accepting a 'raw' ion current signal and producing pressure curve measurants as output. Variations on this have been tried with poor results. It seems that, for this kind of task, simple networks such as the ADALINE can no longer keep up.

Finally, not all data are necessarily in linear relations. Figure 10 is an example of two measurants that seem to indicate rather strongly that a nonlinear relation exists between them. Modified networks have been tried to see whether performance can be improved by passing the inputs through a function, thus making the curve resemble a more linear form. The most important measurants, peak pressure position and magnitude, sometimes improved by as little as 2-3 per cent. This is not a particularly strong case for adding computational cost to the system. It seems that, given the uncertainty in the data and the short segment of curve in question, a linear approximation is best suited to dealing with it.

\section{CONCLUSION}

The results of this research demonstrate the feasibility of bypassing the use of a pressure transducer as a means of in-cylinder data gathering.

Through the use of the ion current signal, manipulated by computationally inexpensive ANNs, the peak pressure position has been predicted to a best resolution of $2.55^{\circ} \mathrm{CA}$ at a sampling rate of one sample per $2^{\circ}$ CA.

Coupled with the robustness and significantly lower cost of the ion-current-sensing apparatus, this performance is indicative of the importance of ion current sensing as a tool in the development of future closed-loop controllers needing to acquire fast cycleper-cycle combustion information.

\section{REFERENCES}

1 Schnauffer, K. Engine cylinder flame propagation studies by new methods. SAE technical paper, 1934.

2 Iinuma, K. Studies of engine combustion processes by ionization current. Bull. of JSME, 1961, 4(4), 352-357.

3 Byttner, S., Rognvaldsson, T., and Wickstrom, N. Estimation of combustion variability using incylinder ionization measurements. SAE technical paper 2001-01-3485, 2001.
4 Yoshiyama, S., Tomita, E., and Hamamoto, Y. Fundamental study on combustion diagnostics using a spark plug as ion probe. SAE technical paper 2000-01-2828, 2000.

5 Shimasaki, Y., Maki, H., Sakaguchi, J., Nishizawa, K., Kato, A., Suzuki, H., Kondo, N., and Yamada, T. Study on combustion monitoring system for formula one engines using ionic current measurement. SAE technical paper 2004-01-1921, 2004.

6 Yoshiyama, S., Tomita, E., and Hamamoto, Y. Fundamental study on combustion diagnostics using a spark plug as ion probe. SAE technical paper 2000-01-2828, 2000.

7 Aithal, S. M., White, A. R., Subramaniam, V. V., Babu, V., and Rizzoni, G. A chemical kinetics model of current signatures in an ionization sensor. http: //www.cis.ohio-state.edu/ saday/894/spark9.doc.

8 Forster, J., Gunther, A., Ketterer, M., and Wald, K. Ion current sensing for spark ignition engines. SAE technical paper 1999-01-0204, 1999.

9 VanDyne, E. A., Burckmyer, C. L., and Wahl A. M. Misfire detection from ionization feedback utilizing the smartfire plasma ignition technology. SAE technical paper 2000-01-1377, 2000.

10 Lunsdstrom, D. and Schagerberg, S. Misfire detection for prechamber SI engines using ion-sensing and rotational speed measurements. SAE technical paper 2001-01-0993, 2001.

11 Raymond, R., Andre, S., and Fabian, M. Local airfuel ratio measurements using the spark plug as an ionization sensor. SAE technical paper 970856, 1997.

12 Devesh, U. and Giorgio, R. AFR control on a single cylinder engine using the ionization current. SAE technical paper 980203, 1998.

13 Balles, E. N., VanDyne, E. A., Wahl, A. M., Ratton, K., and Lai, M. In-cylinder air-fuel ratio approximation using spark gap ionization sensing. SAE technical paper 980166, 1998.

14 Hellring, M., Munther, T., Rognvaldsson, T., Wickstrom, N., Carlsson, C., Larsson, M., and Nytomt, J. Robust AFR estimation using the ion current and neural networks. SAE technical paper 1999-01-1161, 1999.

15 Schneider, D. and Lai, M. D. Real-time air/fuel ratio control in a small SI engine using the ionic current signal. SAE technical paper 1999-01-3323, 1999.

16 Klovmark, H., Rask, P., and Forssell, U. Estimating the air/fuel ratio from gaussian parametrizations of the ionization currents in internal combustion SI engines. SAE technical paper 2000-01-1245, 2000.

17 Ohashi, Y., Fukui, W., Tanabe, F., and Ueda, A. The application of ionic current detection system for the combustion limit control. SAE technical paper 980171, 1998.

18 Bie, T., Ericsson, M., and Rask, P. A novel start algorithm for CNG engines using ion sense technology. SAE technical paper 2000-01-2800, 2000.

19 Eriksson, L., Nielsen, L., and Glavenius, M. Closed loop ignition control by ionization current interpretation. SAE technical paper 970854, 1998. 
20 Asano, M., Kuma, T., Kajitani, M., and Takeuchi, M. Development of new ion current combustion control system. SAE technical paper 980162, 1998.

21 Franke, A., Einewall, P., Johansson, B., and Reinmann, R. Employing an ionization sensor for combustion diagnostics in a lean burn natural gas engine. SAE technical paper 2001-01-0992, 2001.

22 Hellring, M., Munther, T., Rognvaldsson, T., and Wickstrom, N. Spark advance control using the ion current and neural soft sensors. SAE technical paper 1999-01-1162, 1999.

23 Wickstrom, N., Taveniku, M., Linde, A., Larsson, M., and Svensson, B. Estimating peak pressure position and air-fuel ratio using the ionization current and artificial neural networks. In Proceedings of the IEEE Conference on Intelligent transportation systems, Boston, 1997 (IEEE, New York).

24 Saitzkoff, A., Reinmann, R., and Mauss, F. In-cylinder pressure measurements using the spark plug as an ionization sensor. SAE technical paper 970857, 1997.

25 Hellring, M. and Holmberg, U. An ion current based peak-finding algorithm for pressure peak position estimation. SAE technical paper 2000-01-2829, 2000.

26 Hellring, M., Rognvaldsson, T., Wickstrom, N., and Larsson, M. Ion current based pressure peak detection under different air humidity conditions. In Advanced microsystems for automotive applications (AMAA) (Eds S. Krueger and W. Gessner), 2000 (Springer, Berlin).

27 Eriksson, L. Spark-advance control by ion-sensing and interpretation. http://www.vehicular.isy.liu.se/ larer/Projects/main.html.

28 Malaczynski, G. W. and Baker, M. E. Real-time digital signal processing of ionization current for engine diagnostic and control. SAE technical paper 2003-01-1119, 2003.

29 Peron, L., Charlet, A., Higelin, P., Moreau, B., and Burq, J. F. Limitations of ionization current sensors and comparison with cylinder pressure sensors. SAE technical paper 2000-01-2830, 2000.

30 Asano, M., Ito, A., Kuma, T., Kajitani, M., Takeuchi, M., Fukumura, Y., and Izumi, M. Further development of an ion current combustion control system. SAE technical paper 2001-01-0266, 2001.

31 Russ, S., Peet, G., and Stockhausen, W. Measurements of the effect of in-cylinder motion on flame development and cycle-to-cycle variations using an ionization probe head gasket. SAE technical paper 970507, 1997.

32 SMETEC GmbH, http://www.smetec.de. 